\title{
LA GESTIÓN AMBIENTAL DE LOS RESIDUOS SÓLIDOS DE LA TELEFONÍA MÓVIL
}

\section{Victor Manuel Cruz Ornetta}

\section{Resumen}

Las grandes cantidades de residuos provenientes de los teléfonos móviles se han convertido en una preocupación en el ámbito global, es por eso que este estudio tiene como objetivo tomar conocimiento de los elementos necesarios para poder realizar la gestión ambiental de dichos residuos: Para ello se revisó importantes artículos y otros documentos sobre los tóxicos y sus efectos, así como la legislación global y nacional respecto de este tema. Como resultado, se obtuvo el estado del arte del tema incluyendo la magnitud de los residuos sólidos de la telefonía móvil, los tipos de residuos de las baterías de respaldo de las estaciones bases, los tipos de residuos de los teléfonos móviles, la toxicidad de las baterías de respaldo, la toxicidad de teléfonos móviles y sus baterias, los lineamientos internacionales para la gestión de los residuos. Como conclusión, se puede señalar que se ha demostrado que los residuos sólidos de la telefonía móvil tiene una toxicidad ampliamente demostrada y que existen lineamientos internacionales para gestionar los residuos sólidos de la telefonía móvil, los que podrian servir de base para la normativa peruana.

Palabras clave: Residuos teléfonos, baterías teléfonos, baterías estaciones bases, WEEE, residuos electrónicos.

\section{Abstract}

The big amounts of wastes from mobile phones have become a serious concern all over the world, so this is the reason why the objective of this study is to give the necessary elements to carry out the environmental management of these wastes. In order to perform this study it was revised not only some papers and documents on these wastes and their toxicity but also worldwide and national 
legislation on these subjects. As a result it was gotten the state of the art on these subjects including the amount of the solid wastes from mobile telephony, the characterization of wastes from base station, the characterization of wastes from mobile phones and their batteries, the toxicity of base station batteries, the toxicity of mobile phones and their batteries, the international guidelines, and Peruvian legislation for waste management. As a conclusion it could be pointed out that wastes from mobile telephony are very toxic but there are International guidelines to manage them that could be the base for the Peruvian legislation on wastes from mobile telephony.

Key words: Phone waste, phone batteries, base station batteries, WEEE, electronic waste.

\section{INTRODUCCIÓN}

Cada año se generan entre 20 a 50 millones de toneladas de residuos electrónicos alrededor del mundo, constituyendo los residuos sólidos con mayor velocidad de crecimiento, llegando al $1 \%$ del total desechado en el mundo entero (UNEP). Muchos países en desarrollo enfrentan el fantasma de peligrosas montañas de residuos electrónicos con posibles consecuencias negativas para el ambiente y la salud pública.

Debido a procesos de reciclamiento demasiado básicos, muchos contaminantes persistentes bio-acumulativos y tóxicos (PBTs) y metales pesados son liberados de los residuos electrónicos, los que pueden fácilmente acumularse en el cuerpo humano a través de la inhalación de aire contaminado, causando enfermedades vasculares o aún cáncer. Cuando los metales pesados no son adecuadamente tratados, pueden causar daños irreversibles al ambiente, tales como la contaminación del agua

La mejor manera de gestionar los residuos electrónicos debe incluir todo el ciclo de vida del equipo. Durante la fabricación es necesario considerar un diseño de equipo que permita un fácil desensamblaje y reciclaje, para un uso múltiple y prolongado tiempo de vida, evitar el uso de fuertes contaminantes, minimizar el uso de recursos, utilizar las regulaciones y estándares. Al final de la vida útil es necesario realizar una gestión adecuada de los residuos electrónicos.

\section{MATERIALES Y MÉTODOS}

Para cumplir con el objetivo planteado, en este estudio se realizo la revisión de diferentes documentos publicados en el ámbito internacional por organizaciones internacionales y países involucrados en el tema.

\section{RESULTADOS}

El estudio se enfocó en los siguientes temas principales: La magnitud de los residuos sólidos de la telefonía móvil, los tipos de residuos de las baterias de respaldo de las estaciones bases, los tipos de residuos de los teléfonos móviles, la toxicidad de 
las baterias de respaldo, la toxicidad de los teléfonos móviles y sus baterias, los lineamientos internacionales para la gestión de los residuos.

\subsection{La magnitud de los residuos sólidos generados}

Los residuos sólidos originados por los teléfonos móviles se han convertido en uno de los impactos ambientales más importantes de las redes de telefonía móvil ya que la cantidad de teléfonos móviles desechados es proporcional al crecimiento de la red de telefonía móvil que en estos momentos está en el orden de 7000 millones de abonados a nivel mundial [1], 590 millones de abonados en Latinoamérica [2] y más de 30 millones de abonados en el Perú [3]. En la figura 1 se muestran residuos de teléfonos móviles recolectados.

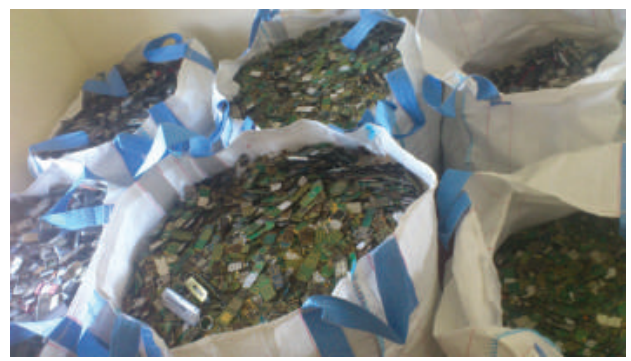

Fig. 1 Residuos de teléfonos móviles

En el ámbito mundial y en el Perú conviven un conjunto de tecnologías que incluyen teléfonos móviles Code Division Multiple Access (CDMA), Time Division Syncronous Code Division Multiple Access (TDSCDMA), Global System Mobile (GSM), Enhanced Data rates for GSM (GSM/EDGE), Wideband Code Division Multiple Access (WCDMA) y Long Term Evolution (LTE) [4]. Tomando en cuenta las di- ferencias tecnológicas entre operadores con teléfonos móviles típicamente bloqueados para cambio de operador debido al contrato, los abonados del Perú en la mayoría de casos [5] y en muchos lugares del mundo, cuando cambian de proveedor de servicios, tienen que cambiar de teléfono móvil y comprar uno nuevo, aún cuando el anterior esté totalmente operativo [6]. En Estados Unidos, de acuerdo a la revista financiera Barron's, el porcentaje anual de usuarios que cambiaban de proveedor de servicios al año 2001 eran aproximadamente $30 \%$ [7].

Debido a las razones mencionadas y al cambio tecnológico constante, el incremento de abonados implica una mayor cantidad de teléfonos móviles y sus accesorios fuera de servicio, estimándose que en el 2009 se producian alrededor de 50 millones de toneladas anuales de residuos electrónicos en el mundo, de los cuales aproximadamente 7000 toneladas corresponden a los desechos de teléfonos móviles en el Perú \% [8].

\subsection{Residuos de las baterias de respaldo de las estaciones bases}

Las baterias de las estaciones base en la mayoría de casos son de plomo $\%$ [9], y pueden tener una vida útil relativamente larga comparadas con las baterias de los teléfonos móviles; pero es necesario incluirlas dentro del plan de gestión ambiental para su disposición final adecuada.

\subsection{Residuos de los teléfonos móviles}

Los diferentes tipos de teléfonos móviles que conviven en el ámbito 
mundial de las diferentes tecnologías que incluyen teléfonos CDMA, TDSCDMA, GSM, WCDMA y LTE tienen una estructura básica muy parecida.

El teléfono móvil es un equipo de abonado que tiene un receptor y un transmisor además de una unidad lógica para la señalización con la estación base. En la figura 2 se puede ver un teléfono móvil de tecnología GSM mostrando algunos de sus componentes.

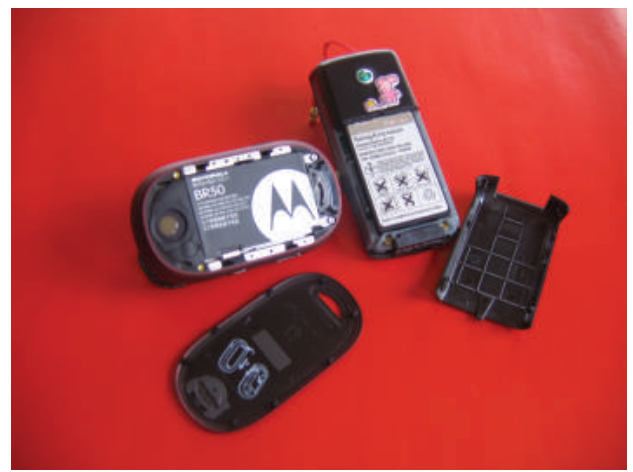

Fig. 2 Teléfono móvil típico mostrando algunos de sus componentes

Los componentes básicos son: tarjeta de circuitos, pantalla de cristal líquido, teclado, antena, parlante, micrófono y carcasa. Como accesorios, se tiene: la batería y su cargador (ver figura 3).

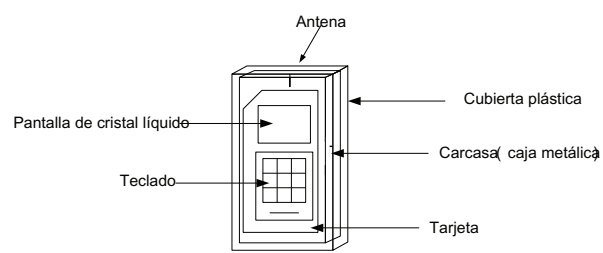

Fig. 3 Partes principales de un teléfono móvil
De acuerdo a estudios realizados por la Deft Technology University de los Países Bajos, la tarjeta de circuitos y la pantalla de cristal líquido, sin considerar los accesorios, son los responsables del 98\% de los impactos ambientales del teléfono móvil en las etapas de producción y reciclaje, lo cual es concordante y complementario con lo que señala la compañía Suiza TCO Development, en el sentido de que la mayoría de los impactos ambientales de los teléfonos móviles provienen de la tarjeta de circuitos, la pantalla de cristal líquido y las baterías [7].

\subsection{Toxicidad de los teléfonos móviles}

Entre las sustancias tóxicas que contienen los teléfonos móviles están los PBTs. Los contaminantes persistentes bio-acumulativos y tóxicos (PBTs) son sustancias relacionadas a numerosos efectos adversos en seres humanos y animales [10], que permanecen en el ambiente por un periodo largo sin degradarse. Los PBTs que forman parte de los teléfonos móviles son el antimonio, arsénico, berilio, cadmio, cobre, plomo níquel y zinc $[11,12]$. De acuerdo a la Agencia de Protección Ambiental de Estados Unidos (EPA), el antimonio, el arsénico, el berilio, el cadmio, el cobre, el plomo, el níquel, el zinc [13] están clasificados dentro del Acta de Respuesta Ambiental Amplia, Compensación y Responsabilidad, químicos sobre los cuales se están haciendo esfuerzos prioritarios para eliminar o reducir su uso o, en el peor de los casos, recuperarlos o reciclarlos. 
El plomo es un metal ampliamente utilizado en los teléfonos móviles principalmente para la soldadura de los componentes electrónicos a los circuitos impresos.

El plomo y el cadmio son bioacumulables en las plantas y animales, introduciéndose a la cadena alimenticia en niveles peligrosos aun cuando se liberan en pequeñas cantidades, pudiendo causar cáncer, desórdenes reproductivos, neurológicos y del desarrollo, y pueden comprometer el sistema inmunológico de los niños $[14,15]$ y contaminar el aire si se incineran o se desechan en grandes cantidades, en botaderos o en rellenos sanitarios.

El berilio contribuye a la dureza, fortaleza, conductividad y resistencia a la corrosión de las aleaciones berilio-cobre (aproximadamente $2 \%$ de berilio), utilizadas en los teléfonos móviles y puede ser un peligro serio para la salud en las instalaciones para manufactura y reciclaje, ya que bajo la forma de polvos o humos de berilio se constituye en uno de los metales más tóxicos a inhalar [16].

El tántalo es un metal precioso altamente resistente a la corrosión y al calor, usado en los condensadores de los teléfonos móviles. De acuerdo a los estudios realizados, su toxicidad es baja debido a su baja solubilidad [17].

El arsénico forma parte de los semiconductores de galio-arsénico, y puede causar daños a los nervios, piel y sistema digestivo [18].

La presencia de cobre en la tarjeta de circuitos incrementa el riesgo de que los retardadores de llamas brominados formen dioxinas y furanos (compuestos orgánicos policlorinados, algunos de los cuales son altamente tóxicos para los animales) [19], si es que se incineran los teléfonos móviles. Adicionalmente, si la combustión se hace a temperaturas relativamente bajas la combustión será incompleta lo que aumentará este riesgo.

Los retardadores de llamas brominados que se incorporan al plástico del teléfono móvil para reducir el riesgo de incendio pueden causar daño al hígado, disfunción de la tiroides y desórdenes del sistema endocrino [20], si los fluidos resultantes se filtran en el suelo o en el agua del subsuelo cuando son dispuestos en botaderos y rellenos sanitarios.

\subsection{Toxicidad de las baterias de las estaciones bases}

Las baterías de respaldo de las estaciones base principalmente son baterías de plomo y tal como se señaló anteriormente el plomo es bioacumulable en las plantas y animales siendo peligroso aun en pequeñas proporciones respecto a los constituyentes del organismo humano. El plomo puede causar cáncer, desórdenes reproductivos, neurológicos y del desarrollo, y puede comprometer el sistema inmunológico de los niños y contaminar el aire, la tierra o el agua [14], si se incineran o se desechan inadecuadamente en botaderos o en rellenos sanitarios.

\subsection{Toxicidad de las baterias para teléfonos móviles}

La baterías más usadas son las de níquel-metal-hidrido y las de litio-ión. 
Las baterias de níquel-metalhidrido, tienen entre 30 a 50\% de níquel y compuestos de níquel, de 5 a $20 \%$ de zinc y de 2.5 a $8 \%$ de cobalto y compuestos de cobalto.

Las baterías de litio-ión tienen 2 a $15 \%$ de cobre y menos de $25 \%$ de cobalto y compuestos de cobalto.

\subsection{Lineamientos internacionales} para la gestión de los residuos sólidos Iniciativa "Mobile Phone Partnership Initiative (MPPI)" de la Convención de Basilea [21], cuyos objetivos son:

- La reducción de movimientos transfronterizos de residuos peligrosos y otros residuos sujetos a la Convención de Basilea

- La prevención y minimización de la generación de residuos peligrosos

- La activa promoción de la transferencia y uso de tecnologias más limpias

Para el logro de estos objetivos se han constituido cuatro proyectos

- Proyecto 1: Restauración y reuso de teléfonos móviles de los teléfonos móviles usados.

- Proyecto 2: Recolección y movimiento fronterizo de teléfonos móviles usados.

- Proyecto 3: Recuperación y reciclaje de teléfonos móviles al final de su uso útil.

- Proyecto 4: Toma de conciencia en la consideraciones de diseño y entrenamiento.

\section{Directivas de la Unión Europea}

Waste of electric and electronic equipment (WEEE) (desechos de equipos eléctrico y electrónico) [22].

El propósito de esta directiva es contribuir a la producción y consumo sostenible y como primera prioridad la prevención de WEEE, adicionalmente, mediante el reuso y reciclado y otras formas de recuperación de residuos, de tal manera de reducir la disposición de residuos y contribuir al uso eficiente de los recursos y la recuperación de las materias primas secundarias valiosas También busca mejorar el rendimiento ambiental de todos los operadores directamente involucrados en el ciclo de vida de los WEEE.

Restrictions of hazard substances (RoHS) (Restricción de sustancias peligrosas) [23].

Sus objetivos, son:

- Reducir los niveles de sustancias peligrosas usadas en los aparatos electrónicos nuevos, habiendo sido la meta la eliminación de sustancias peligrosas, tales como mercurio, plomo, cadmio, cromo y los retardadores de llamas brominados, para el 01 de julio de 2006.

- Evitar/ minimizar mediante la estandarización

Las empresas ambientalmente conscientes tienen programas de minimización de basura electrónica, pero tales programas son dificiles de iniciar y gestionar, los costos adicionales pueden desanimarlas. Como compañias individuales el impacto puede ser pequeño, pues se necesita crear una masa crítica y actuar pronto, la regulación es compleja y toma mucho tiempo.

Resoluciones y recomendaciones de la Unión Internacional de Telecomunicaciones (UIT). 
Resolución 79 de la Unión Internacional de Telecomunicaciones UITT sobre desechos electrónicos [24] Aprobada en la Asamblea Mundial de Normalización de las Telecomunicaciones (Dubai, 2012), cuyos objetivos son:

- Contribuir a mitigar el impacto negativo de los desechos electrónicos en el medio ambiente y la salud;

- Continuar y fortalecer el desarrollo de las actividades de la UIT, en lo que se refiere a la manipulación y el control de los desechos electrónicos de los equipos de TIC y los métodos de tratamiento de los mismos, a través de:

- Mejores prácticas;

- Recomendaciones, metodologías y otras publicaciones;

- Orientación para los responsables políticos;

- Ayuda a los países en desarrollo, que son los países que más sufren los peligros de los desechos electrónicos, sin ser los más responsables;

- Colaboración con todas las partes interesadas pertinentes.

Recommendation ITU-T L1000 Universal power adapter and charger solution for mobile terminals and other hand-held ICT devices [25]

Prevé los requisitos de alto nivel relativos al adaptador y cargador universal de energía que reducirán el número de adaptadores y cargadores de energía elaborados y reciclados puesto que se aplicarán a un número mayor de dispositivos, los que tendrán un ciclo de vida útil más prolongado. Esta solución también tiene por finalidad reducir el consumo de energía. El ciclo de vida más prolongado y la posibilidad de evitar la duplicación de dispositivos reducen la demanda de materias primas y los residuos. El adaptador y cargador universal de energía ha sido diseñado para la gran mayoría de terminales móviles y otros dispositivos TIC.

Recommendation ITU-T L-1100 Procedure for recycling rare metals in information and communication technology goods [26]

Describe los procedimientos para el reciclaje de metales raros en bienes de tecnología de la información y la comunicación (TIC). También define un formato de comunicación para el suministro de información sobre el reciclaje de metales raros contenidos en bienes de TIC.

\section{CONCLUSIONES Y RECOMENDACIONES}

Se ha cumplido con los objetivos del estudio ya que se ha demostrado que los residuos sólidos de la telefonía móvil conforman una fuente importante de los residuos electrónicos cuya toxicidad es ampliamente demostrada por estudios realizados principalmente por la OMS. Así mismo se ha conseguido información de las iniciativas internacionales para gestionar los residuos sólidos de la telefonía móvil. Se recomienda que el Perú implemente su normatividad respecto de la gestión de los residuos sólidos de la telefonía móvil que crecen constantemente. 


\section{REFERENCIAS BIBLIOGRÁFICAS}

ERICSSON. Ericsson Mobility Report [Internet]. Estocolmo: Ericsson Junio 2013 [citado 30 de septiembre de 2013]. Disponible en http://www.ericsson. $\mathrm{com} / \mathrm{res} /$ docs/2013/ericsson-mobility-report-june-2013.pdf

GSMA. Observatorio Móvil de América Latina - Resumen Ejecutivo. Londres: GSMA 2013 [citado 30 de septiembre de 2013] Disponible en http://www.gsma. $\mathrm{com} /$ publicpolicy/wp-content/uploads/2012/04/latammoesspa-2.pdf

ORGANISMO SUPERVISOR DE LA INVERSIÓN PRIVADA EN TELECOMUNICACIONES [Internet]. Indicadores del servicio móvil. Lima: OSIPTEL [citado 30 de septiembre de 2013]. Disponible en http://www.osiptel. gob.pe/WebsiteAjax/WebFormgeneral/sector/wfrm_Consulta_Informacion_ Estadisticas .aspx ?CodInfo $=13478 \%$ CodSubCat $=864 \&$ TituloInformacion $=2 . \% 20$ Indicadores $\% 20 \mathrm{del} \% 20$ Servicio $\% 20 \mathrm{M} \% \mathrm{c} \% \mathrm{~b} 3 v \mathrm{vil}$ DescripcionInformacion=

WIKIPEDIA [Internet]. Anexo: Empresas de telefonía móvil de Latinoamérica. Wikipedia [citado 30 de septiembre de 2013]. Disponible en http:// es.wikipedia.org/wiki/Anexo:Empresas_de_telefon\%C3\%ADa_m\%C3\%B3vil_ de_Latinoam\%C3\%A9rica

ORGANISMO SUPERVISOR DE LA INVERSIÓN PRIVADA EN TELECOMUNICACIONES [Internet]. Campañas de comunicación. Campañas de comunicación -2009. Sitio web Portabilidad Numérica. Informate sobre la Portabilidad Numérica. Preguntas frecuentes. Lima: OSIPTEL [citado 30 de septiembre de 2013]. Disponible en:

http://www.osiptel.gob.pe/WebSiteAjax/archivos/comunicaciones / portabilidad/portabilidad_pf2.htm

MINISTERIO DE INDUSTRIA, ENERGÍA Y TURISMO DE ESPAÑA. Telefonía móvil [Internet]. Cambio de operador. Madrid: MINETUR [citado 30 de septiembre de 2013]. Disponible en http://www.usuariosteleco.es/Derechos/ TelefoniaMovil/Paginas/cambio-operador.aspx

FISHBEIN B. Waste in the Wireless World: The Challenge of Cell Phones [Internet]. Nueva York :INFORM; 2002 [citado 30 de septiembre de 2013]. Disponible en:

http:/ / informinc.org/reportpdfs/wp/WasteintheWirelessWorld.pdf

UNESCO. Los residuos electrónicos un desafio para la Sociedad del Conocimiento en América Latina y el Caribe [Internet]. Montevideo: UNESCO; 2010 [citado 30 de septiembre de 2013]. Disponible en:

http://www.unesco.org.uy/ci/fileadmin/comunicacion-informacion/ LibroE-Basura-web.pdf

SCHARNHOST, Wolfram. Life Cycle Assessment of Mobile Telephone networks, with focus on the End-Of-Life Phase. The International Journal of Life Cycle Assessment. Julio 2006 11; 4: 290-291. 
AGENCIA DE PROTECCIÓN AMBIENTAL DE LOS ESTADOS UNIDOS [Internet] . Persistent Bioaccumulative and Toxic (PBT) Chemical Program. About PBTs. Washington: EPA; 2013 [citado 30 de septiembre de 2013]. Disponible en http://www.epa.gov/opptintr/pbt/pubs/aboutpbt.htm

LIM SR, Schoenung JM. Toxicity potentials from waste cellular phones, and a waste management policy integrating consumer, corporate, and government responsibilities. Waste Manag. Agosto 2010;30(8-9):1653-60

NNOROM I, Osibanjo O. Toxicity characterization of waste mobile phone plastics. Journal of Hazardous Materials. 2009; 161: 183-188

Agencia de Protección Ambiental de los Estados Unidos.

Consolidated List of Chemicals Subject to the Emergency Planning and Community Right- To-Know Act (EPCRA), Comprehensive Environmental Response, Compensation and Liability Act (CERCLA) and Section 112(r) of the Clean Air Act [Internet]. Washington: EPA; 2011 [citado 30 de septiembre de 2013]. Disponible en:

http://www.epa.gov/osweroe1/docs/chem/list_of_lists_revised_7_26_2011. pdf

ORGANIZACIÓN PANAMERICANA DE LA SALUD [Internet]. . Evaluación del riesgo por la exposición a Plomo. Washington: OPS; 1999 [citado 30 de septiembre de 2013]. Disponible en http:/ / www.bvsde.paho.org/tutorial/casos/caso3/plom. html

ORGANIZACIÓN MUNDIAL DE LA SALUD. Cadmium in Drinking-water [Internet].. Ginebra: OMS; 2011 [citado 30 de septiembre de 2013]. Disponible en http://www.who.int/water_sanitation_health/dwq/chemicals/cadmium.pdf

ORGANIZACIÓN MUNDIAL DE LA SALUD. Beryllium and beryllium compounds [Internet]. Ginebra: OMS; 2011 [citado 30 de septiembre de 2013]. Disponible en http://www.who.int/ipcs/publications/cicad/en/cicad32.pdf.

ORGANIZACIÓN INTERNACIONAL DEL TRABAJO [Internet]. Tantalum. Ginebra: OIT; [citado 30 de septiembre de 2013]. Disponible en

http: / /www.ilo.org/oshenc/part-ix/metals-chemical-properties-andtoxicity/item/190-tantalum

ORGANIZACIÓN MUNDIAL DE LA SALUD [Internet]. Arsenic. Ginebra: OMS; 2012 [citado 30 de septiembre de 2013]. Disponible en http://www.who.int/ mediacentre/factsheets/fs372/en/.

ORGANIZACIÓN MUNDIAL DE LA SALUD. Copper in Drinking-water [Internet]. Ginebra: OMS; 2004 [citado 30 de septiembre de 2013]. Disponible en http://www.who.int/water_sanitation_health/dwq/chemicals/copper.pdf

WIKIPEDIA. Retardantes de llamas bromados. Wikipedia; 2010 [citado 30 de septiembre de 2013]. Disponible en http://es.wikipedia.org/w/index.php?title= Especial:Libro\&bookcmd=download\&collection_id=16f25e8b9cf5e90d\&writer=r 18return_to=Retardantes+de+llama+bromados 
UNITED NATIONS ENVIRONMENT PROGRAMME. Mobile Phone Partnership Initiative [Internet]. Ginebra: UNEP; 2010 [citado 30 de septiembre de 2013]. Disponible en http://archive.basel.int/industry/mppi/gdfd30Jun2010.pdf

WASTE ELECTRICAL AND ELECTRONIC EQUIPMENT (WEEE). Directive 2012/19/EU (Official Journal of the European Union, Estrasburgo 4 de julio de 2012).

Restricciones a la utilización de determinadas sustancias peligrosas en aparatos eléctricos y electrónicos. Directiva 2011/65/UE (Diario Oficial de la Unión Europea, Estrasburgo 8 de junio de 2011).

UNIÓN INTERNACIONAL DE TELECOMUNICACIONES. Resolución 79 - Función de las telecomunicaciones/tecnologías de la información y la comunicación en el tratamiento y el control de residuos electrónicos de equipos de telecomunicaciones y tecnologías de la información, y métodos para su procesamiento. Ginebra: UIT; 2012.

UNIÓN INTERNACIONAL DE TELECOMUNICACIONES. Recommendation ITU-T L.1000 - Universal power adapter and charger solution for mobile terminals and other hand-held ICT devices. Ginebra: UIT; 2011.

UNIÓN INTERNACIONAL DE TELECOMUNICACIONES. Recommendation ITU-T L.1100- Procedure for recycling rare metals in information and communication technology goods. Ginebra: UIT; 2012. 